\title{
Quantitative evaluation of die casting surface defect severity by analyzing surface height
}

\author{
*Yan Xu, Naoya Hirata and Koichi Anzai \\ Department of Metallurgy, Graduate School of Engineering, Tohoku University, Sendai 980-8579, Japan
}

\begin{abstract}
It is necessary in factories to assess the severity of the surface defects of castings, as a slight surface defect will be taken as qualified when it brings no bad effect or it can be removed by the subsequent processing. In practical production, professional technicians visually inspect the surface defect severity according to their individual experience. Therefore, it is difficult for them to maintain the same standard and accuracy in the subjective, tedious and labor-intensive work. Recently, image processing techniques based on optical images have been applied to achieve better accuracy and high efficiency. Unfortunately, optical images cannot directly quantify surface depth, which works as a crucial factor in the practical assessment of surface defect severity. The surface roughness evaluation algorithm, which takes into account of both area and depth information of the assessed surface, was applied to directly characterize surface defect severity based on surface asperity rather than optical image. The results using standard casting pieces show that surface defect severity has no apparent dependence on surface roughness. However, the subsequent results show that the root-mean-squared-deviation (RMSD) of surface gradient of flow line defects positively correlates with the increase of defect severity. The other types of defect do not present such tendency. Thus, practical workpieces with flow line defects on the surface were used to verify the universality of this tendency. The results show that surface roughness of an unqualified workpiece is larger than that of a qualified workpiece after surface slope adjustment, but presents no obvious coincidence before the adjustment. In contrast, the RMSD of an unqualified workpiece, no matter before or after the adjustment, is larger than that of a qualified one.
\end{abstract}

Key words: die casting; surface defect; surface roughness

CLC numbers: TP391.99 Document code: A Article ID: 1672-6421(2017)05-339-07

$\mathrm{V}$ arious types of surface defects of die casting products may occur due to the complex situation inside the die. The evaluation and classification of surface defects on die castings have long been an important and challenging issue. Traditionally, visual inspection depending on technicians' individual experience has been used in factories to examine whether the surface defects are acceptable or not. However, this calls for high requirement on the technicians' skill. Even professional technicians will find it difficult to balance the conflict between efficiency and accuracy, since the work is so subjective, tedious and monotonous.

Image processing technology provides a viable alternative to automatically detect the defects and evaluate the defect severity. Wong ${ }^{[1]}$ et al. adopted fuzzy logic algorithm to surface images after image

\section{*Yan Xu}

Male, Ph.D. candidate in Tohoku university. His research interests mainly focus on the quantitative analysis for microstructure of die castings and compounds using mathematical morphology.

E-mail: yan.xu.t2@dc.tohoku.ac.jp

Received: 2017-08-14; Accepted: 2017-09-22 processing to automatically distinguish the quench crack, mechanical crack and hole on the surface of castings. Defect area, perimeter, length, etc. are basic parameters in fuzzy logic algorithm, in which new parameters like radius ratio, axis ratio and approximate area were calculated to characterize the shape feature of defect area. Świłło ${ }^{[2-3]}$ et al. developed a surface defect inspecting machine for die castings with image processing algorithms based on modified Laplacian of Gaussian edge detection method to recognize defects with different shapes and sizes. Most of the relevant works were carried out by analyzing the optical images taken from the object surfaces and tried to classify surface defects by calculating shape features like area, perimeter, length, etc. However, defect depth (defect height for blisters) also plays an essential role in evaluating surface defect severity. Zhang ${ }^{[4]}$ et al. also tried to analyze the surface defect severity using shape features achieved from image analysis. The combining usage of these features highly improves classifying precision. Most of the relevant works were carried out by analyzing the optical images taken from the object surfaces and tried to classify surface defects 
by calculating shape features like area, perimeter, length, etc. However, defect depth (defect height for blisters) from the bottom (top for blisters) to the expected surface level of sound castings also plays an essential role in evaluating surface defect severity. An evaluation method generally considering defect length, area and depth is desirable. Surface roughness ${ }^{[7]}$ is such a conventional concept. It reflects the surface asperity by calculating surface height variations.

In this study, the surface roughness of standard die casting pieces for surface defects were calculated based on the surface height measured by measuring devices. The correlation of surface roughness and root mean squared deviation of surface gradient to the surface defect severity level was investigated. In the subsequent stage, surface defect on the practical workpieces were analyzed to verify the correlation. The influence of surface slope was also discussed.

\section{Standard samples and surface height measurement}

\subsection{Standard samples}

The samples comprise two groups. One is the standard pieces group obtained from reference [5] and the other is practical workpieces group from a foundry. Table 1 shows the standard pieces group including five types of surface defects, which are flow line, cold shut, shrinkage cavity, blister and dent. All of the pieces are classified into 4 severity levels ranking from level A (slight) to level D (severe). It should be noted that the shrinkage cavity samples are contaminated by cold shut defects at severity level A, B and D.

\subsection{Measurement of surface height}

Surface height of standard casting pieces was measured with a VR-30 3-dimensional shape measuring device with a resolution of $23.362 \mu \mathrm{m} /$ pixel. The measuring zone was a rectangle with sizes of $1200 \times 768$ (pixel $\times$ pixel). The measured results were saved as csv files.

\section{Surface asperity of standard casting pieces}

\subsection{Surface roughness}

There are many algorithms calculating roughness for surface. Generally, the two algorithms depicted in Eq. (1) and Eq. (2)

Table 1: Standard surface defect pieces in reference [10]

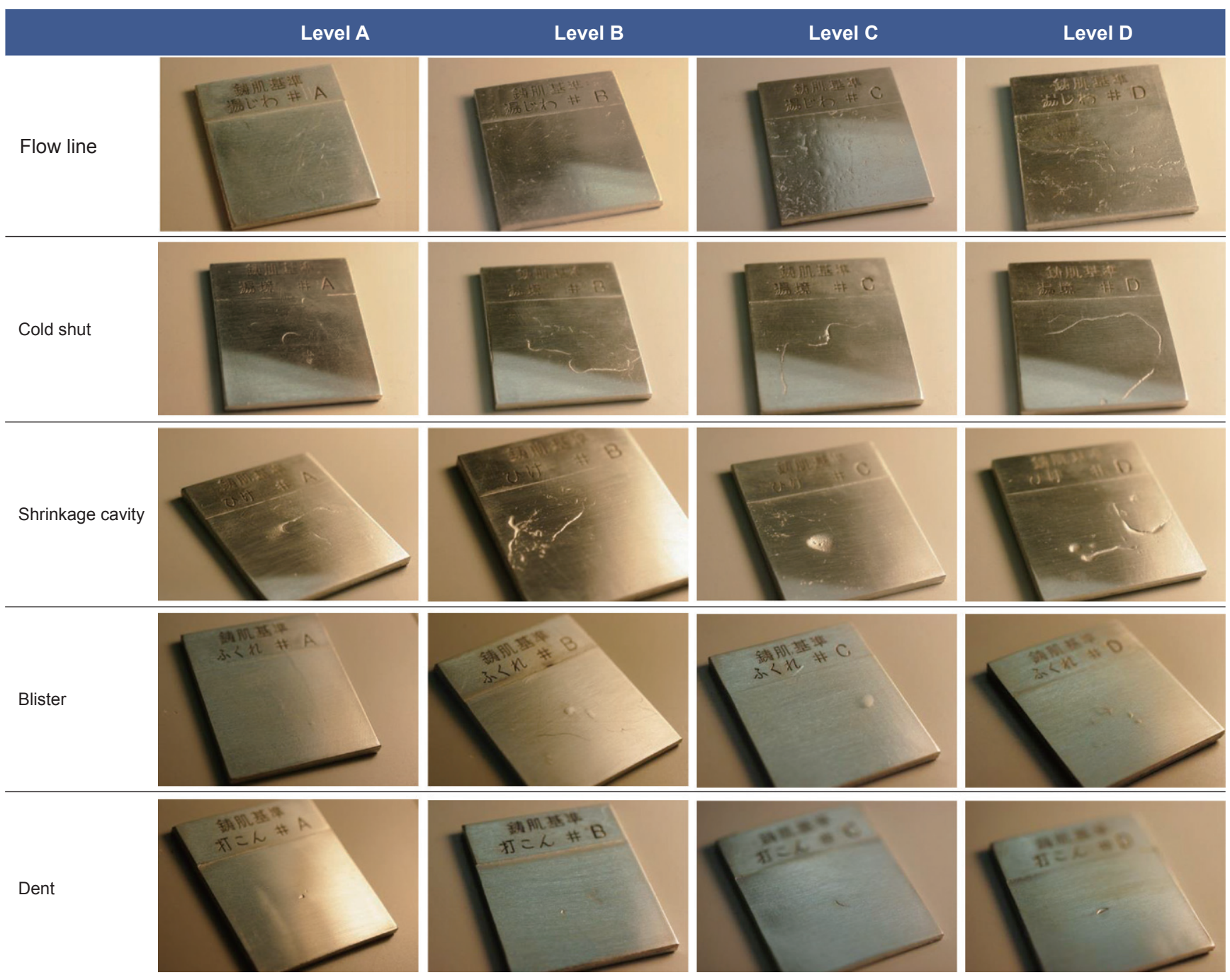


are the most commonly used. They are the arithmetic mean and root mean squared deviation (RMSD) of the difference between surface height and mean surface height.

$$
\begin{gathered}
R_{a}=\frac{1}{N} \sum_{i=1}^{N}\left|h_{i}-\bar{h}\right| \\
R_{q}=\sqrt{\frac{1}{N} \sum_{i=1}^{N}\left(h_{i}-\bar{h}\right)^{2}}
\end{gathered}
$$

where, $N$ is the total number of pixels, $h_{i}(\mu \mathrm{m})$ is the surface height value at pixel $i$ and $h(\mu \mathrm{m})$ is the mean height. Figure 1 illustrates the calculation of $R_{a}(\mu \mathrm{m})$ for a one dimensional surface. Firstly, the mean height is calculated. In the next step, the average absolute deviation between the surface profile and mean height is also achieved and denoted as $R_{a}$. The surface roughness is called $R_{q}(\mu \mathrm{m})$ when the root-mean-squareddeviation (RMSD) of surface height is calculated.

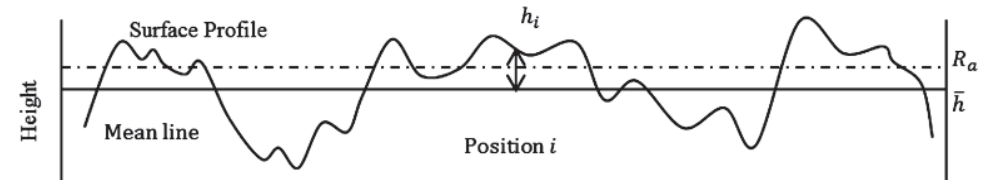

Fig. 1: Illustration of surface roughness calculation for one dimensional surface height

Figure 2 shows the application results of the two surface roughness algorithms on standard casting pieces. The two algorithms produced quite similar results. In both figures, the surface roughness does not show clear relationship with defect severity. The surface roughness of flow line samples first falls down when the severity progresses from level A to level B. Afterwards, it steadily grows up with the severity level increasing to level D. Opposite to the tendency of flow line samples, the cold shut samples firstly increase dramatically

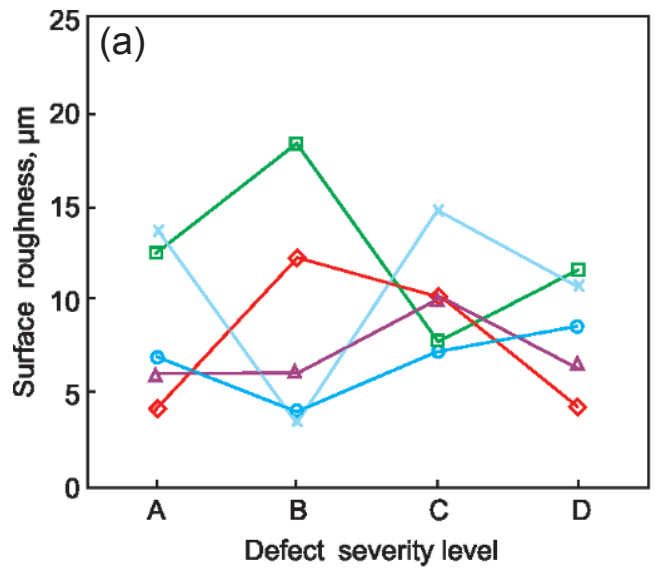

$\multimap$ Flow line $\triangleleft$ Cold shut $\because$ Shrinkage cavity

Fig. 2: Surface roughness results: (a) $R_{a}$ and (b) $R_{q}$

\subsection{Surface gradient}

The severity of surface defects on the standard casting pieces has no monotonic correlation to the surface height frequency. Thus, surface gradient was computed to investigate the correlation. Surface gradient is another commonly used parameter to characterize surface asperity. It has a close correlation with surface height variation in the surface defect areas. It can be calculated with Eq. (3) ${ }^{[6]}$, where, $h$ $(\mu \mathrm{m})$ is the surface height, $g_{x}\left(\mu \mathrm{m} \cdot \mu \mathrm{m}^{-1}\right)$ and $g_{y}\left(\mu \mathrm{m} \cdot \mu \mathrm{m}^{-1}\right)$ are the gradient in horizontal and vertical directions. In this study, only the magnitude of the gradient, given by Eq. (4), is considered for the reason that the dispersion of surface gradient direction is not necessary for analyzing the surface asperity here. from severity level A to B, however, followed by a successive declination from level $\mathrm{B}$ to level $\mathrm{D}$. The surface roughness of blister samples increases smoothly from severity level A to B and C. However, the severest level D sample presents quite low surface roughness, even lower than that of level A sample. The other two defect samples, shrinkage cavity and dent, oscillate when severity level upgraded from A to D. Generally, the surface roughness of standard samples presents no clear monotonic correlations to surface defect severity.

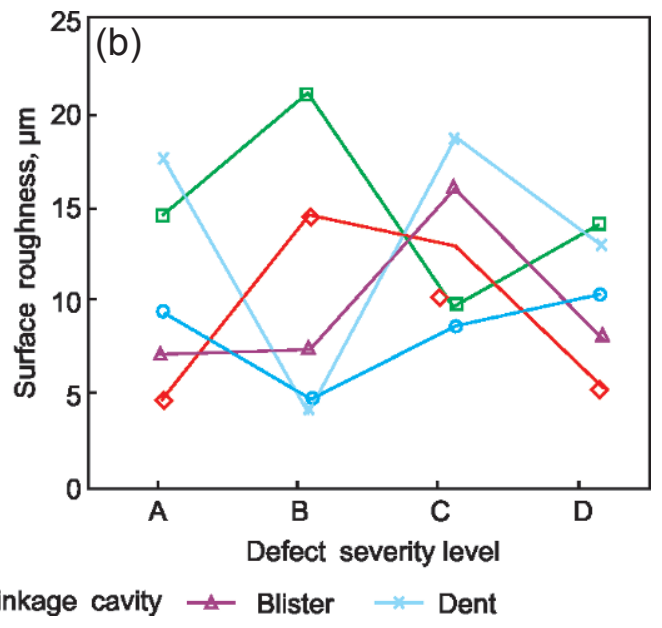

$$
\begin{aligned}
& \nabla h=\left[\begin{array}{l}
g_{x} \\
g_{y}
\end{array}\right]=\left[\begin{array}{l}
\frac{\partial h}{\partial x} \\
\frac{\partial h}{\partial y}
\end{array}\right] \\
& g=\sqrt{\left(g_{x}\right)^{2}+\left(g_{y}\right)^{2}}
\end{aligned}
$$

The distribution frequency of surface gradient of standard casting pieces was calculated with Eq. (3) and Eq. (4) and shown in Fig. 3. Here, the interval between maximum and minimum surface gradients was evenly divided into 20 categories and the numbers of pixels in each category were counted and divided by the total pixel number to calculate the frequency histogram. We counted the numbers of pixels in each piece and divided them by the total pixel number to calculate the distribution frequency. 

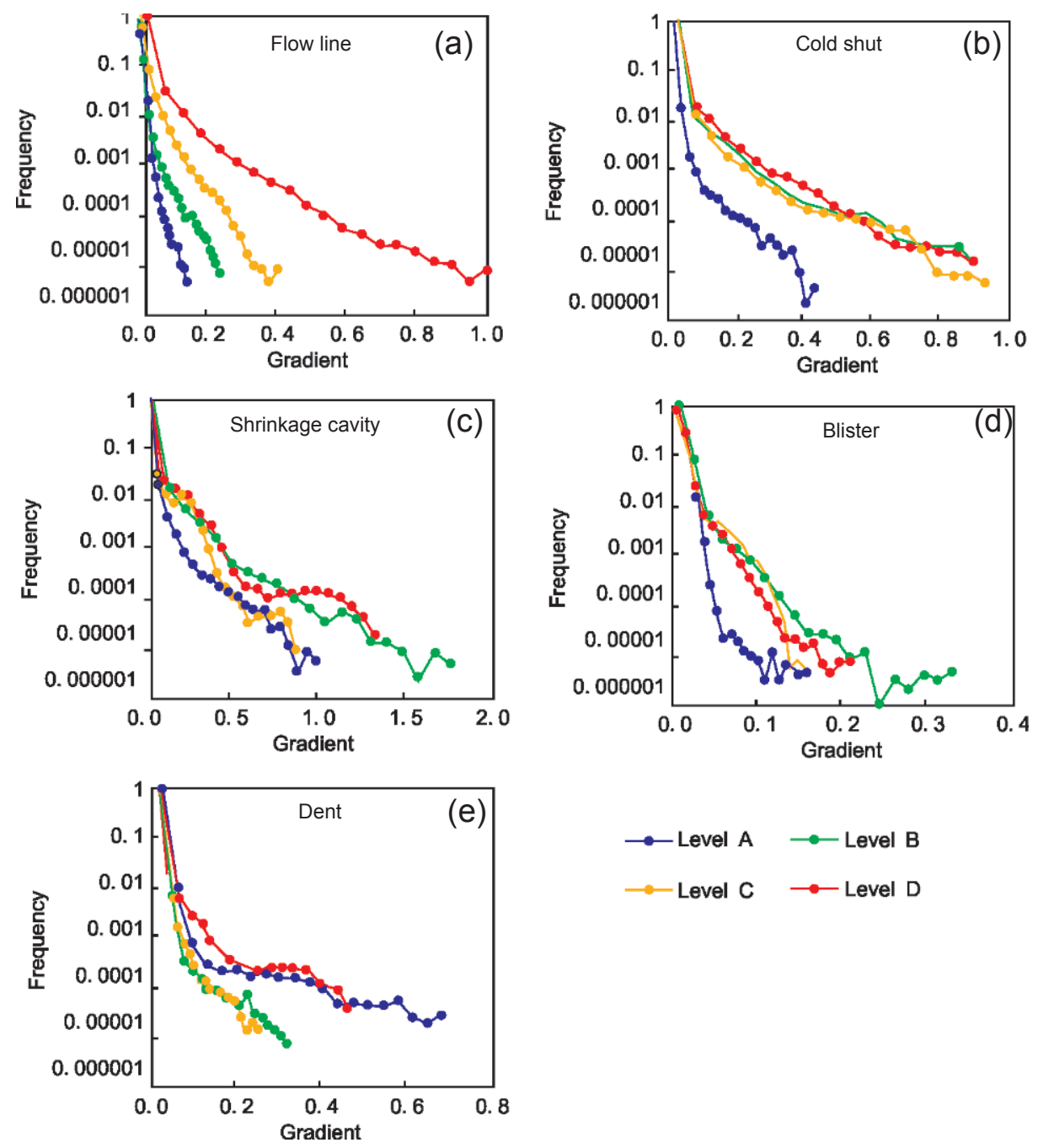

Fig. 3: Surface gradient distribution of standard samples: (a) flow line, (b) cold shut, (c) shrinkage cavity, (d) blister and (e) dent

Polylines were used to demonstrate the variation trend of frequency instead of rectangles to avoid overlaps. In this study, the standard samples were manufactured into a flat sheet shape and measured on a flat platform. Thus, the peaks of the polylines concentrated to 0 . This indicated that there was no surface slope on the surfaces of standard samples. In contrast, those of the other types of defects [shown in Fig. 3(b) to Fig. 3 (e)] did not present such tendency.

RMSD of surface gradient was calculated with Eq. (5):

$$
\sigma=\sqrt{\frac{1}{N} \sum_{i=1}^{N}\left(g_{i}-\bar{g}\right)^{2}}
$$

where, $\sigma$ is the RMSD of surface gradient, $N$ is the total pixel amount, $g_{i}$ is the surface gradient at pixel $i$, and $\bar{g}$ is the average of surface gradient. $\bar{g}$ can be expressed as $\bar{g}=\frac{1}{N} \sum_{i=1}^{N} g_{i}$

Figure 4 shows the RMSD of surface gradient for each type of standard samples. The shrinkage cavity and flow line defects present a monotonic increase tendency with the defect severity level progressed from A to D. In the case of shrinkage cavity, the value of RMSD dramatically increases when the defect severity progresses from level A to level B and level C to level D. The increase is gentle from B to C. However, the frequency polylines

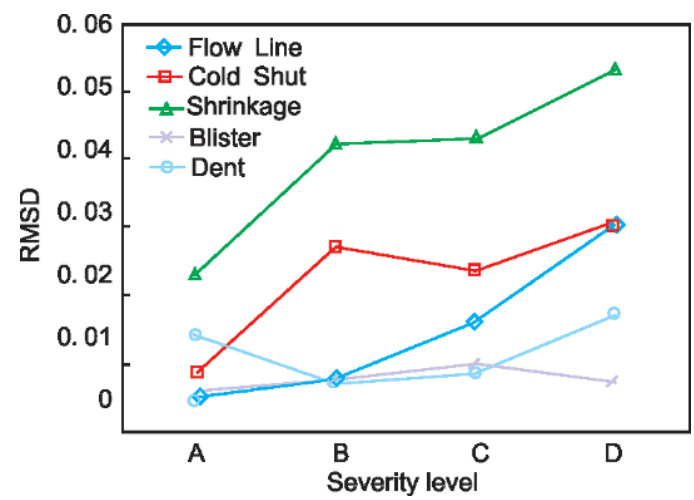

Fig. 4: RMSD of standard samples surface gradient

in Fig. 3(b) are not observed with clear distribution in sequence of severity level. As denoted previously, the shrinkage cavity samples were contaminated by cold shut defects at severity level A, B and D. It is difficult to tell the influence of cold shut defect on the RMSD of surface gradient of shrinkage cavity samples. Thus, the monotonic relationship between RMSD of surface gradient and defect severity level was not reliable. In the case of flow line, the RMSD also shows positive correlation to defect severity, although the increasing rate from level A to level $\mathrm{B}$ is small. This coincided with the surface gradient frequency 
histogram in Fig. 3(a), in which the polylines of severe flow line samples distributed at right side and those of slight flow line samples located at left side in order. The flow line samples were not contaminated by other types of defects. Therefore, the monotonic correlation was reliable. The RMSD of other types of defects shows no monotonic relationship to defect severity.

\section{Verification using practical products}

Figure 5 shows the surface of the practical workpiece samples taken by an optical microscope. There are flow lines on the surface of clutch case workpieces sampled from actual products in a foundry. The 18 specimens were evenly selected from 3 quality levels, which were unqualified, uncertain and qualified, respectively. Literally, unqualified workpieces were those having severe defects that make them fail to match the quality requirement. In contrast, qualified ones are those having slight defects that do not have bad effect on expected performance or can be eliminated by subsequent processing. The samples were marked as uncertain workpieces if technicians felt difficult to determine their severity. Surface height of practical workpieces was measured using the VHX-2000 device with a resolution of $2.122 \mu \mathrm{m}$. The observed zone was a rectangle of size $1600 \times 1200$ (pixel $\times$ pixel). Apparently, the observed zone is very small and thus the surface curvature was neglected in this study.
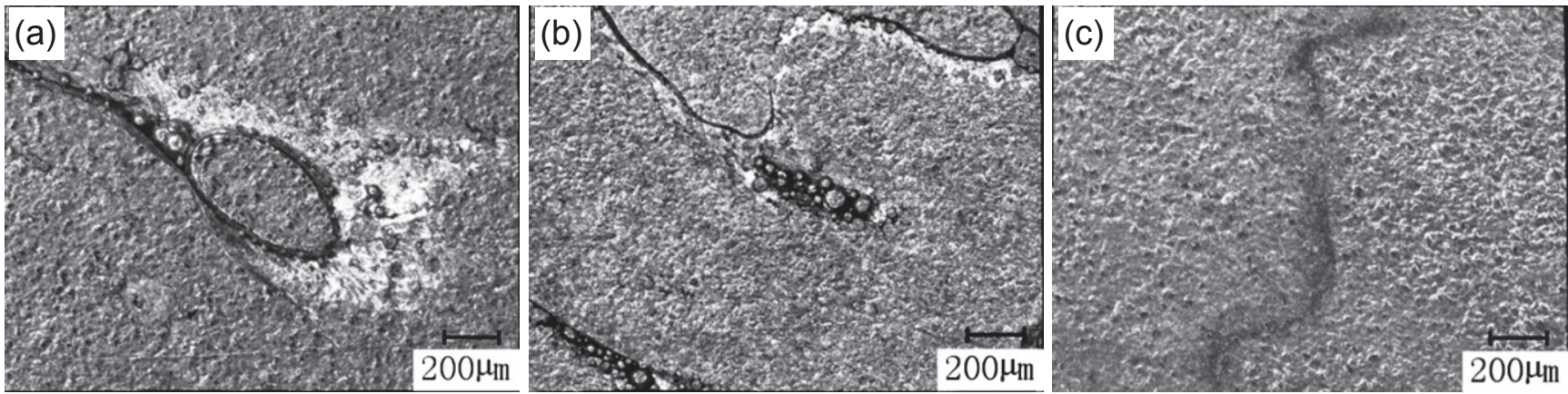

Fig. 5: Examples of flow line defects on surface of practical clutch case castings: (a) unqualified, (b) uncertain, (c) qualified

Different with standard samples with flat surface, the surface of practical workpieces usually accompanied with a large surface slope owing to the complex geometry of the workpieces. Therefore, adjustment had to be made first to reduce the influence of surface slope before calculating surface roughness. We used the surface normal vector to construct a reference plane to correct surface slope. The procedure is depicted as follows: assume that the surface of casting is in a three dimensional Euclidean space $R^{3}$, where xy-plane denotes the horizontal and vertical axis and $z$-axis indicates the surface height variation direction. A plane passing $(0,0,0)$ and perpendicular to the

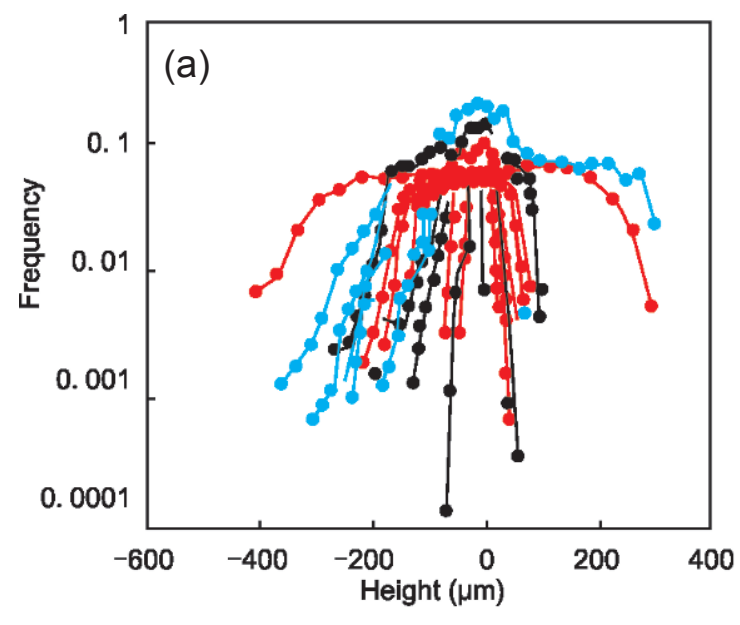

$\longrightarrow$ Unqualified normal vector of the surface can be constructed. This is the referring plane and the height difference between it and the actual surface is taken as corrected surface height. Normal vectors of the surfaces were estimated using average normal vector. It is given in Eq. (6), where, $\vec{n}_{\text {surface }}$ is the average normal vector of the casting surface, $\vec{n}$ is the normal vector at certain pixel, and $S$ is the surface area.

Figure 6 shows the surface height distribution of practical samples before [Fig. 6(a)] and after [Fig. 6(b)] the surface slope adjustment. It can be seen that before the adjustment, frequency polylines entangled together and presented no clear dependence

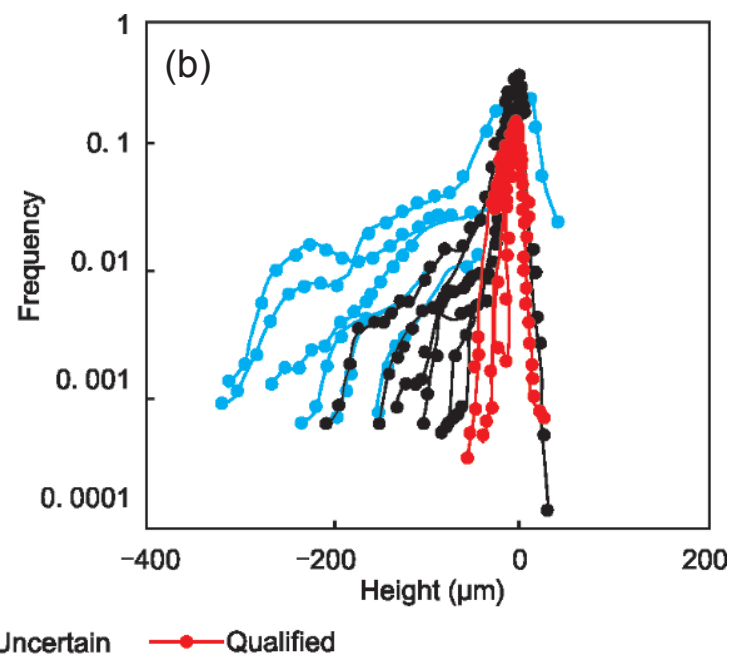

Fig. 6: Surface height distribution before (a) and after (b) surface slope adjustment 
on defect severity. In contrast, the results after adjustment showed much clearer coincidence. The polylines of qualified samples distributed in a very small range, while those of uncertain and unqualified samples distributed in a larger range successively.

\subsection{Surface roughness}

Figure 7 shows the surface roughness results of practical samples before and after surface slope adjustment. It has been addressed previously that $R_{a}$ and $R_{q}$ produced quite similar results in Fig. 2. Therefore, we only calculated $R_{a}$ for the practical samples. Before eliminating the influence of surface slope, surface roughness of the qualified sample was $142 \mu \mathrm{m}$. It was even larger than any unqualified sample. It was hard to tell the correlation between surface roughness and defect severity.

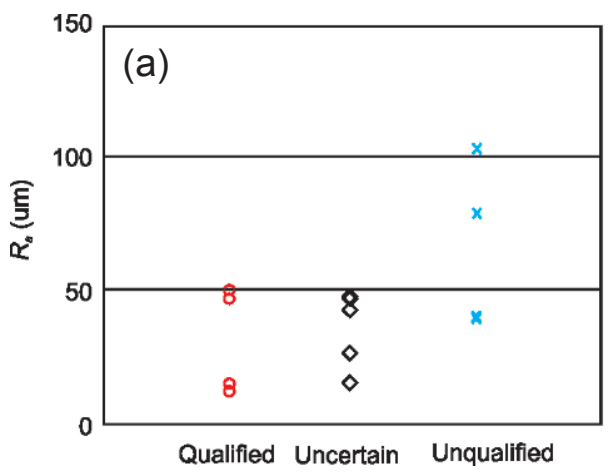

In contrast, the relationship between them became clear after the adjustment. The surface roughness of a qualified sample was smaller than that of an unqualified sample, whereas the surface roughness of standard samples showed no monotonic correlation with the upgrade of defect severity, as shown in Fig. 2. This is because considering that there was no contamination of other types of surface defect on the surfaces of standard samples, we only observed a single flow line defect on practical samples but more than one flow line defects on the standard samples. Surface roughness is a statistic absolute average of surface height variations, which means that the surface with many swallow flow lines might have larger surface roughness than that with fewer but deeper flow lines. Therefore, the surface roughness result differed with the sequence of severity level of standard samples.

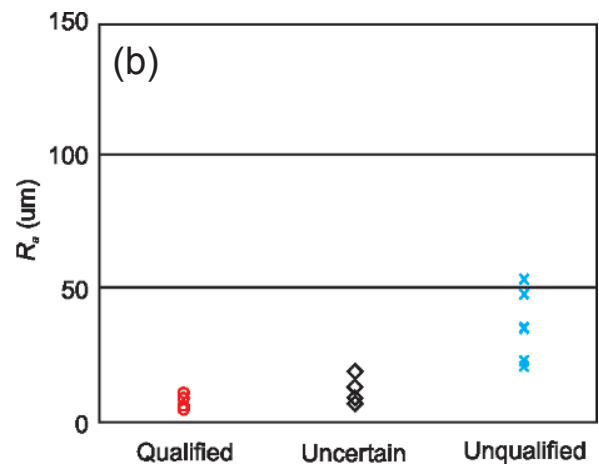

Fig. 7: Surface roughness of practical clutch case samples before (a) and after (b) surface slope adjustment

\subsection{Surface gradient}

Figure 8 shows the surface gradient distribution of the practical samples. The polyline peaks of samples after adjustment concentrated close to gradient 0 , whereas the peaks of samples before adjustment deviated a little from 0 . It should be noted that the gradient of a surface was its slope. Thus, the peak of a surface gradient frequency polyline before adjustment located at the surface slope magnification, as shown in Fig. 8(a). After the adjustment, all the peaks moved to 0 , as the surface slope had been eliminated. This proved that the adjustment reduced the surface slope well. Similar to surface gradient distribution of standard

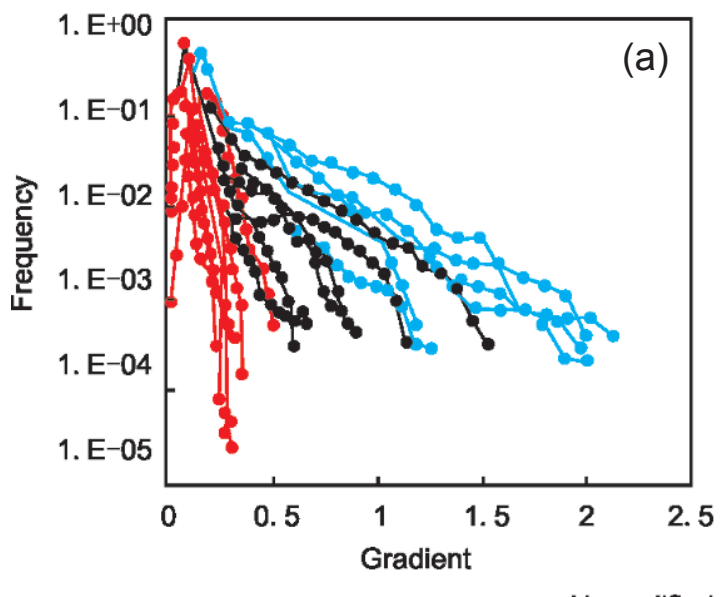

$\longrightarrow$ Unqualified samples, qualified samples also distributed in a smaller range and uncertain and unqualified samples successively distributed in larger ranges. The surface gradient values of qualified samples dispersed within the interval $[0,0.5]$, while those of the other samples, no matter uncertain or unqualified, exceeded this interval.

Figure 9 shows the RMSD results of practical samples before and after the surface slope adjustment. It can be seen that RMSD had little variation after the adjustment. This is because surface slope shifted the peaks of gradient distribution polyline, but had little influence on the polyline size and shape. All of the qualified practical samples had very small RMSD

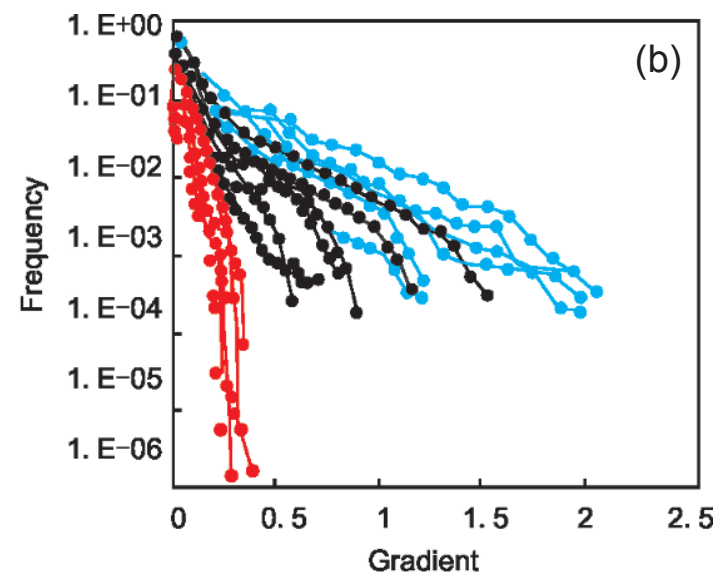

Fig. 8: Surface gradient distribution of practical samples before (a) and after (b) surface slope adjustment 


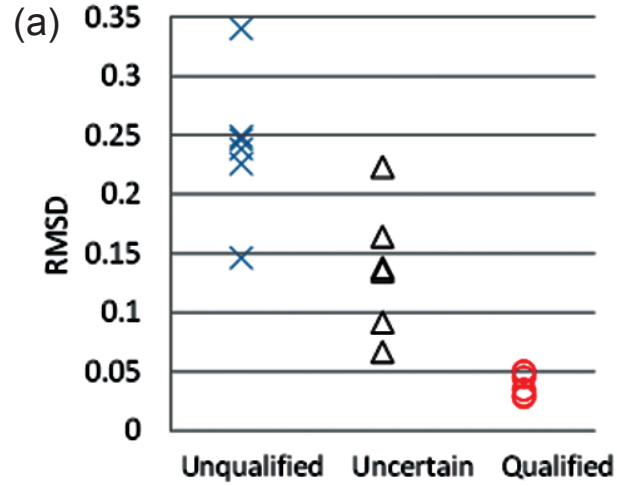

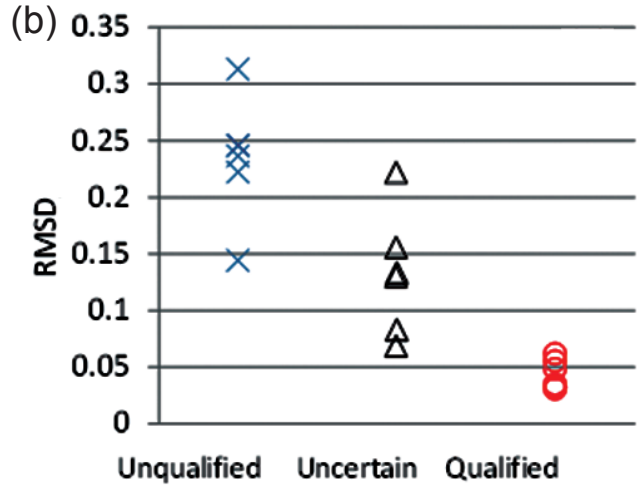

Fig. 9: RMSD of practical samples before (a) and after (b) surface slope adjustment

of surface gradient, which was no larger than 0.07 before the adjustment and 0.05 after the adjustment. In contrast, RMSD of the unqualified ones was over 0.14 no matter with or without adjustment. This coincided with Fig. 8, where the polylines of unqualified samples were at the larger range and those of qualified samples concentrated in the smaller range. RMSD value positively correlated to the flow line defect severity. This monotonic relationship between RMSD of surface gradient and defect severity provides a potential method to automatically, objectively and quantitatively evaluate flow line defect severity. In the first step, a RMSD threshold can be obtained by analyzing samples classified by human visual inspection. Subsequently, the flow line defects can be automatically assessed by calculating RMSD of surface gradient. Samples with a larger RMSD than the threshold can be assessed as unqualified.

\section{Conclusions}

Quantitative evaluation of die casting surface defects by analyzing surface asperity was carried out. Firstly, standard samples were investigated using surface roughness algorithms and surface gradient algorithms. The results showed that, although the surface roughness had little monotonic correlation to defect severity, the RMSD of surface gradient of flow line defect samples presented coincidence with the upgrade of defect severity. However, the other types of defects did not show clear relationship. Practical samples were also investigated subsequently to verify the effectiveness of the method. Surface slope was observed on the surfaces of practical samples due to their complex geometry. Therefore, surface slope was eliminated with average normal vector method in the first stage. Afterwards, surface roughness, surface gradient frequency and RMSD of surface gradient were calculated. The results showed that practical samples with severer flow line defect had large surface roughness after surface slope adjustment. Also, the RMSD of surface gradient of practical samples positively correlated to flow line defect severity.

\section{References}

[1] Wong B K, Elliott M P, Rapley C W. Automatic casting surface defect recognition and classification. In: Proc. IEE Colloq. Appl. Mach. Vis., May 24, 1995: 10/1-10/5.

[2] Świłło S J, Perzyk M. Automatic inspection of surface defects in die castings after machining. Archives of Foundry Engineering, 2011, 11(3): 231-236.

[3] Świłło S J, Perzyk M, Surface casting defects inspection using vision system and neural network techniques. Archives of Foundry Engineering, 2013, 13(4): 103-106.

[4] Zhang Xiang, Krewet C, Kuhlenkötter B. Automatic classification of defects on the product surface in grinding and polishing. International Journal of Machine Tools \& Manufacture, 2006, 46(1): 59-69.

[5] Japan Die Casting Association. Die Casting Standard, Quality series. Tokyo: Futaba Layout Press, 2012.

[6] Gonzalez R C, Woods R E. Digital Image Processing. USA: Prentice Hall, 2008.

[7] BS EN ISO 4287, Geometrical Product Specification (GPS). Surface texture - Profile method: Terms, Definitions and Surface Texture Parameters, 2000 